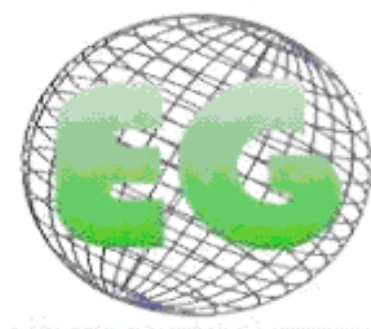

ISSN 1695-6141 N 27
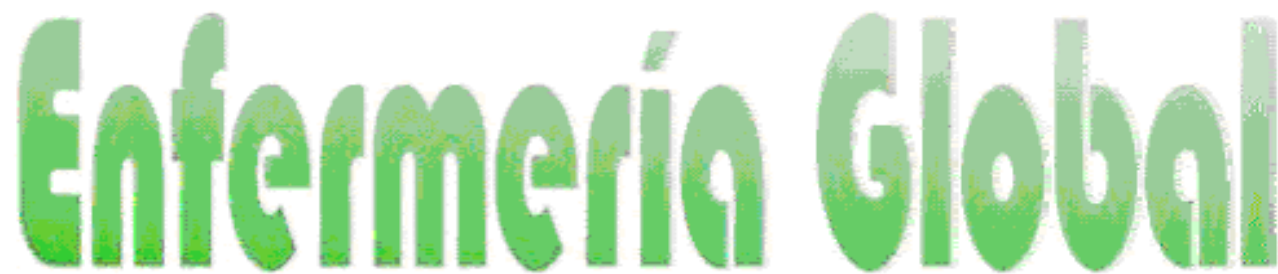

Revista electrónica trimestral de Enfermería

Julio 2012

www.um.es/egloball

\title{
Correlaciones de variables del Inventario de Burnout de Maslach en profesionales de urgencia hospitalaria
}

Correlações de variáveis do Inventário de Burnout de Maslach em profissionais de emergência hospitalar

\author{
${ }^{*}$ Ritter, RS., ${ }^{* *}$ Fernandes Stumm, EM., ${ }^{* * *}$ Kirchner, RM., ${ }^{* * *}$ Schmidt Piovesan \\ Rosanelli, CL., ${ }^{* * * * * U b e s s i, ~ L D . ~}$
}

\begin{abstract}
*Enfermera. Pos-graduada en Enfermería Cardiológica y Terapia Intensiva por la Universidad Regional del Noroeste del Estado de Rio Grande do Sul - Unijuí, ljuí-RS/Brasil. E-mail: renata.ritter87@yahoo.com.br **Enfermera, Máster en Administración por la UFRGS/Brasil, docente en Unijuí, orientadora. ***Graduada en Matemáticas, Doctora en Ingeniería Eléctrica por la PUC/RJ, docente em la Universidad Federal de Santa Maria, colaboradora. "****Enfermera, Máster en Educación en las Ciencias, docente de Unijuí, colaboradora. *****Psicóloga, estudiante de Enfermería, mestranda en Educación en las Ciencias por la Unijuí, colaboradora.
\end{abstract}

Palabras clave: Servicios Médicos de Emergencia; Burnout; profesionales de la Salud; estrés ocupacional.

Palavras-chave: Serviços Médicos de Emergência; Burnout; profissionais de Saúde; estresse ocupacional. Keywords: Emergency services; Burnout; health care professionals; occupational stress

\section{RESUMEN}

El Servicio de Urgencias es un entorno que expone a los profesionales a los trastornos psicológicos. La investigación buscó caracterizar los profesionales sanitarios que trabajan en una Unidad de Urgencias de un hospital de tamaño IV del noroeste de Rio Grande do Sul, así como correlacionar variables del Síndrome de Burnout. Es descriptiva, cuantitativa, desarrollada en 2007, con 38 profesionales. Los instrumentos de recolección de datos fueron el Maslach Burnout Inventory (MBI) y datos sociodemográficos. Se utilizó SPSS y estadística descriptiva. El perfil es 57,9\% mujeres, $30-40$ años, $60,5 \%$ casados, con hijos, $70,3 \%$ tienen educación secundaria, el 2,7\% graduación y el 27,0\% de especialización de postgrado. 34,2\% tienen 3,5 años en la profesión y 55,3\% 3,5 años en el Departamento de Emergencia. En cuanto a las dimensiones del síndrome, hay decenas de baja por agotamiento emocional, despersonalización e incompetencia, mostrando bajo nivel de Burnout. El contexto de Trabajo obtuvo puntuaciones elevadas. Se encontraron correlaciones significativas entre "edad", "desgaste emocional" e "incompetencia", entre "tiempo de actuación en emergencia" "incompetencia"," entre el "tiempo de trabajo en situaciones de emergencia" y "desarrollo de la carrera", y entre "desgaste emocional" e "incompetencia"; Despersonalización con Incompetencia y Desarrollo de la carrera; Incompetencia con Estructura Organizacional, Desarrollo de la Carrera y Relaciones Interpersonales; Estructura Organizacional con Sobrecarga de Trabajo (cuantitativo), Sobrecarga de Trabajo (cualitativo) y Relaciones Interpersonales; Sobrecarga de Trabajo (cuantitativo) con Relaciones Interpersonales. La 
producción de más conocimiento puede ayudar en el cotidiano de estos profesionales y estimular a los investigadores a la continuidad de estudios.

\section{RESUMO}

A Unidade de Emergência é um ambiente que expõe os profissionais ao sofrimento psíquico. A pesquisa objetiva caracterizar profissionais da saúde que atuam em uma Unidade de Emergência de um hospital porte IV do noroeste do Rio Grande do Sul, bem como correlacionar variáveis da Síndrome de Burnout. É descritiva, quantitativa, desenvolvida em 2007, com 38 profissionais. Os instrumentos de coleta de dados foram o Inventário de Burnout de Maslach (MBI) e dados sociodemográficos. Utilizou-se SPSS e estatística descritiva. O perfil é $57,9 \%$ feminino, $30-40$ anos de idade, $60,5 \%$ casado, $70,3 \%$ com filhos, $70,3 \%$ possuem ensino médio, 2,7\% graduação e 27,0\% especialização. 34,2\% atuam há 3,5 anos na profissão e 55,3 \% há 3,5 anos na Emergência. Quanto às dimensões da síndrome, observam-se escores baixos para Desgaste Emocional, Despersonalização e Incompetência, demonstrando baixo nível de Burnout. O Contexto do Trabalho apresentou escores elevados. Há correlações significativas entre a "idade", "desgaste emocional" e "incompetência", entre "tempo de atuação em emergência" e "desenvolvimento da carreira", bem como entre Desgaste Emocional e Incompetência; Despersonalização com Incompetência e Desenvolvimento da Carreira; Incompetência com Estrutura Organizacional, Desenvolvimento da Carreira e Relações Interpessoais; Estrutura Organizacional com Sobrecarga de Trabalho (quantitativo), Sobrecarga de Trabalho (qualitativo) e Relações Interpessoais; Sobrecarga de Trabalho (quantitativo) com Relações Interpessoais. A produção de mais conhecimento pode contribuir no cotidiano destes profissionais e instigar pesquisadores à continuidade de estudos.

\section{ABSTRACT}

The emergency service is an environment that exposes the health care professionals to psychological diseases. The research looked for the characterization of the health care professionals who work at an emergency unit in a size IV hospital from the northwest of Rio Grande do Sul and to correlate Burnout Syndrome variables. This research is descriptive, quantitative and it was developed in 2007, with 38 professionals. The tools used for the recording of the data were the Maslach Burnout Inventory (MBI) and the socio-demographic data. A SPSS and descriptive statistics were used. The profile is $57,9 \%$ women, $30-40$ years old, $60,5 \%$ married, with children, $70,3 \%$ have studied secondary education, the $2,7 \%$ got graduated and the $27,0 \%$ got the post grade specialization. 34, 2\% have worked for 3, 5 years in the profession and $55,3 \%$ have worked for 3,5 years at the Emergency Department. Regarding the dimensions of the syndrome, there are tens of sick leaves due to emotional exhaustion, depersonalization and incompetence, showing a low level of Burmout. The context of work got high scores. Significant correlations were found among "age", "emotional deterioration" and "incompetence", between "time of action at emergency" and "incompetence", between the "time of work in emergency situations" and "development of the race", and between "emotional exhaustion" and "incompetence"; Depersonalization with Incompetence and Development of the Race; Incompetence with the Organizational Structure, Development of the Race and Interpersonal Relations; Organizational Structure with Work Overload (quantitative) with Interpersonal Relations. The production of more knowledge may help the daily routine of these professionals and it also may stimulate the researchers to carry on with their studies.

\section{INTRODUCCIÓN}

En las últimas décadas el mundo del trabajo ha sufrido innumerables transformaciones. Se trata de un proceso histórico que se desarrolla, se intensifica y se hace más complejo a lo largo de los años. En este sentido, Saviani ${ }^{1}$ afirma que el acto de actuar sobre la naturaleza transformándola conforme a las necesidades humanas se denomina "trabajo". El autor puntualiza que la esencia humana es el trabajo, o sea, el ser humano se reconoce y es reconocido por la sociedad y por el trabajo que desarrolla.

Al referirse al cotidiano de los profesionales que actúan en el cuidado de individuos en situaciones de emergencia y, en este caso, destacan las Unidades de Emergencia, las UTIs, puede decirse que estos están expuestos a condiciones y ritmos de trabajo extenuantes, pues se enfrentan directamente con lo imprevisto, el sufrimiento y la muerte, situaciones estas susceptibles de generar estrés. 
En cuanto al estrés ocupacional, Barros; Tironi; Sobrinho ${ }^{2}$ destacan que la primera reacción de estrés relacionada con el trabajo consiste en estar exhausto, agotamiento, sobrecarga física y mental y dificultades de relación. Paralelo a esto, se constata el distanciamiento ylo indiferencia y, consecuentemente, la ineficiencia en el trabajo. Diferentes autores dirigen sus estudios al análisis y comprensión del significado del sufrimiento humano ligado al trabajo, especialmente el Síndrome de Burnout. ${ }^{3}$

El Síndrome de Burnout es un problema psicosocial que está afectando a profesionales de diversas áreas, entre ellas, a los de la salud. Carlotto; Câmara ${ }^{4}$ dicen que el Burnout es un proceso formado por tres dimensiones relacionadas pero independientes una de la otra: Desgaste Emocional (DE), Despersonalización (DD) e Incompetencia. El Desgaste Emocional se caracteriza por la falta o carencia de energía, entusiasmo y por sentimiento de agotamiento de recursos. Los trabajadores creen que no están en condiciones de gastar energía en la atención del usuario y demás personas como hacían anteriormente. La Despersonalización hace que los profesionales traten a los usuarios, colegas y a la organización como objetos, hasta desarrollar insensibilidad emocional. La Incompetencia se revela por una tendencia de los trabajadores a autoevaluarse de forma negativa. Se sienten infelices, insatisfechos y con sentimiento de baja realización profesional.

El Burnout tiende a afectar a trabajadores motivados, que se entregan totalmente al trabajo como reacción al estrés laboral y acaban por entrar en colapso. Ellos invierten mucha más energía de la que reciben en términos de reconocimiento y resultados ${ }^{5}$. En este sentido, destacar a los profesionales de servicios que actúan directamente con usuarios en actividades de cuidado, como profesionales de salud y educación, policiales y agentes penitenciarios.

Investigaciones han mostrado la incidencia mundial de individuos afectados por el síndrome de Burnout entre médicos y enfermeras, revelando altos índices de Desgaste Emocional, Despersonalización y sentimiento de Incompetencia, constatándose principalmente entre trabajadores de salud que actúan en unidades de cuidados de emergencia, tales como UTIs y Unidades de Urgencias, conforme mencionado anteriormente ${ }^{6}$. El Síndrome de Burnout está presente en estas unidades implicado directamente con las condiciones y características específicas del trabajo, con las consecuentes demandas psicológicas y emocionales en los respectivos profesionales que allí actúan.

La busca incesante de la excelencia, muchas veces sobrepasa los límites del trabajador y acaba en enfermedad. Importa destacar que la personalidad, la cultura, los valores y principios del ser humano influyen considerablemente, tanto en las percepciones como en las respuestas a los estresores vivenciados en el ambiente de trabajo. El estrés es experimentado por cada individuo, de forma única, o sea, lo que para uno puede ser traumático, para otro puede ser solo un desafío. De esta forma, la subjetividad interfiere en las respuestas al estrés y, por tanto, está intimamente relacionada con el desarrollo del Síndrome de Burnout, que deriva del estrés crónico.

Cuidar es la esencia de la enfermería y, así, se destaca la necesidad de ampliar la concienciación de los profesionales referentes a cuidar de sí mismos, mirando la reducción de los efectos nocivos del estrés, con repercusiones positivas en su salud y en la calidad de vida y mayor resolutividad en la asistencia a los pacientes y en los resultados organizacionales.

Considerando lo expuesto, se busca con esta investigación caracterizar al equipo de profesionales de la salud que trabaja en una Unidad de Urgencia de un hospital porte IV del noroeste de Rio Grande do Sul, así como correlacionar las variables del Síndrome de Burnout. 


\section{MATERIAL Y MÉTODOS}

El presente artículo se realizó a partir de resultados obtenidos en una investigación descriptiva, de enfoque cuantitativo, desarrollada en 2007, en una Unidad de Ugencia de un hospital porte IV de la región noroeste del estado de Rio Grande do Sul, considerado referencia en salud. El referido hospital es filantrópico, atiende pacientes en diferentes niveles de complejidad y la Unidad de Ugencia, lugar de la investigación, cuenta con 24 camas de observación. El equipo se compone de 03 enfermeros, 27 técnicos en enfermería, 03 auxiliares de enfermería y 08 médicos. Integraron la investigación todos los profesionales de la salud (38) que trabajaban en la referida unidad, y que, voluntariamente, aceptaron participar de la misma.

En cuanto a los aspectos éticos que abarca una investigación con seres humanos, todos se cumplieron. El proyecto de investigación fue dirigido y aprobado por el Comitê de Ética en Investigación de la UNIJUI, bajo Parecer Consubstanciado № 094/2007. La colecta de datos se realizón en el mes de julio de 2007, con los siguientes instrumentos: Inventario de Burnout de Maslach-MBI, validado por Lautert ${ }^{7}$, compuesto de 52 (cincuenta y dos) preguntas fechadas, con grado de intensidad/frecuencia para la respuesta y datos sociodemográficos, este creado y testado por las investigadoras.

El "Inventario de Burnout" va acompañado de una escala de conversión, que permite clasificar las respuestas de los investigados en: Desgaste Emocional, Despersonalización e Incompetencia. Hay también una escala de conversión referente al Contexto de Trabajo. En el análisis cuantitativo de las variables que caracterizan el Síndrome de Burnout, se utilizó una escala del tipo Likert, con valores entre 1 y 5 para las respuestas de los profesionales, considerando 1 (nunca), 2 (algunas veces al año), 3 (algunas veces al mes), 4 (algunas veces a la semana) y 5 (diariamente). A partir de esta puntuación fue posible calcular media, desvío estandard y coeficiente de variación.

Los datos se analizaron con el software estadístico SPSS, estadística descriptiva y presentados en tablas. Utilizado el Coeficiente de Correlaciónde Pearson en que $\rho=1$ significa una correlación perfecta positiva entre dos variables, mientras que $\rho=-1$ significa una correlación perfecta negativa entre las variables, esto es, si una aumenta, la otra siempre disminuye. En resumen, " $\rho$ " adquiere su significancia conforme su valor, o sea, "0.70" para más o para menos indica una fuerte correlación, "0.30 a 0.7 " positivo o negativo indica correlación moderada y "0 a 0.30 " significa débil correlación.

\section{RESULTADOS}

En lo que se refiere a las características sociodemográficas de los profesionales investigados, en la Tabla 1 se constata similitud entre el sexo femenino $(57,9 \%)$ y el masculino $(42,1 \%)$. Se trata de una población joven, en plena fase productiva, casada $(60,5 \%)$ y la mayoría con hijos. En cuanto a la escolaridad, se evidencia que la mayoría cursó la enseñanza media y un porcentual próximo de $30 \%$ es especialista. En cuanto a la actividad profesional, hay predominio de técnicos en enfermería, seguido de médicos, enfermeros y auxiliares de enfermería. 


\begin{tabular}{|c|c|c|}
\hline Característica & $\mathrm{N}$ & $\%$ \\
\hline \multicolumn{3}{|l|}{ Sexo } \\
\hline Feminino & 22 & 57,9 \\
\hline Masculino & 16 & 42,1 \\
\hline \multicolumn{3}{|l|}{ Escolaridade } \\
\hline Ensino médio & 26 & 70,3 \\
\hline Graduação & 1 & 2,7 \\
\hline Especialização & 10 & 27,0 \\
\hline \multicolumn{3}{|l|}{ Estado Civil } \\
\hline Casado & 23 & 60,5 \\
\hline Solteiro & 13 & 34,2 \\
\hline Separado & 2 & 5,3 \\
\hline \multicolumn{3}{|l|}{ Idade ${ }^{*}$} \\
\hline 20 --- 30 anos & 10 & 27,0 \\
\hline 30 --- 40 anos & 16 & 43,3 \\
\hline 40 --- 50anos & 8 & 21,6 \\
\hline 50 anos ou mais & 3 & 8,1 \\
\hline \multicolumn{3}{|l|}{ Atividade Profissional ${ }^{\star}$} \\
\hline Técnica em enfermagem & 24 & 64,9 \\
\hline Médico(a) & 7 & 18,9 \\
\hline Enfermeiro(a) & 3 & 8,1 \\
\hline Auxiliar de enfermagem & 3 & 8,1 \\
\hline \multicolumn{3}{|l|}{ Filhos* } \\
\hline Sim & 26 & 70,3 \\
\hline Não & 11 & 29,7 \\
\hline
\end{tabular}

*um pesquisado não respondeu

Secuencialmente, la Tabla 2 presenta la caracterización del tiempo de actuación de los profesionales investigados. En ella se constata que 50\% del equipo está formado de 1 a 11 años incompletos, más de $60 \%$ trabaja en el $\mathrm{HCl}$ de 1 a 11 años, así como en la Unidad de Emergencia, por eso el porcentual es más elevado, o sea, más del $70 \%$. 


\begin{tabular}{|c|c|c|}
\hline Tempo & $\mathrm{N}$ & $\%$ \\
\hline \multicolumn{3}{|l|}{ De profissão } \\
\hline Menos de 1 ano & 4 & 10,5 \\
\hline $1 \mid--6$ anos & 13 & 34,2 \\
\hline $6 \mid---11$ anos & 6 & 15,8 \\
\hline $11 \mid---16$ anos & 4 & 10,5 \\
\hline 16 anos ou mais & 11 & 29,0 \\
\hline \multicolumn{3}{|l|}{ Atuação no $\mathrm{HCl}$ de ljuí } \\
\hline Menos de 1 ano & 4 & 10,5 \\
\hline $1 \mid--6$ anos & 17 & 44,7 \\
\hline $6 \mid---11$ anos & 7 & 18,4 \\
\hline $11 \mid---16$ anos & 4 & 10,5 \\
\hline 16 anos ou mais & 6 & 15,8 \\
\hline \multicolumn{3}{|c|}{ Atuação em Unidade de } \\
\hline Emergência & 5 & 13,2 \\
\hline Menos de 1 ano & 21 & 55,3 \\
\hline $1 \mid--6$ anos & 7 & 18,4 \\
\hline $6 \mid---11$ anos & 3 & 7,9 \\
\hline 11 --- 16 anos & 2 & 5,2 \\
\hline 16 anos ou mais & & \\
\hline
\end{tabular}

En la secuencia (ver Tabla 3), se presentan los resultados referentes a utilización del Inventario de Burnout de Maslach (MBI) en los profesionales de la Unidad de Urgencia investigada. Al analizar las medias obtenidas en la escala de conversión, se observan indicadores relativamente bajos en las tres dimensiones del síndrome, mostrando bajo nivel de Burnout en los investigados.

Tabela 3- Estatística descritiva da escala de conversão do Inventário de Burnout de Maslach

\begin{tabular}{lc}
\hline Escala de conversão & Média \pm Desvio Padrão \\
\hline Desgaste Emocional & $2,08 \pm 0,35$ \\
Despersonalização & $1,78 \pm 0,37$ \\
Incompetência & $1,44 \pm 0,41$ \\
\hline Ambigüidade de Funções & $1,29 \pm 0,40$ \\
Estrutura Organizacional & $2,96 \pm 0,49$ \\
Sobrecarga de Trabalho & \\
(quantitativo) & $3,43 \pm 1,37$ \\
Sobrecarga de Trabalho & \\
(qualitativo) & $2,45 \pm 0,28$ \\
Desenvolvimento da Carreira & $3,16 \pm 1,18$ \\
Relações Interpessoais & $2,60 \pm 0,49$ \\
\hline
\end{tabular}


En cuanto a Ambigüedad de Funciones en el contexto del trabajo, se constata una media relativamente baja, lo que puede considerarse un resultado positivo, toda vez que la misma surge cuando el trabajador no tiene claro el papel que desempeña. En lo que se refiere a la Estructura Organizacional, se observa una media considerablemente elevada, generalmente, como consecuencia de los impactos de la organización del trabajo sobre la salud, o bienestar físico, mental y social de los trabajadores.

Paralelo a esto, se observa media elevada en la categoría Sobrecarga de Trabajo (cuantitativo) y media limítrofe en la categoría Sobrecarga de Trabajo (cualitativo), evidenciando acentuada heterogeneidad en las respuestas de los investigados, lo que significa que algunos se sienten sobrecargados en cuanto los demás consiguen manejarse tranquilamente con las actividades en su cotidiano en el trabajo. La categoría Desarrollo de la Carrera también reveló una media relativamente alta, en cuanto que en la de Relaciones Interpersonales se constata pequeña elevación de su media, resultado considerado positivo.

Dando continuidad a la presentación de los resultados obtenidos en esta investigación, la Tabla 4 presenta la correlación de la edad, tiempo de profesión y de actuación en emergencia con las variables de la escala de conversión del MBI. En esta se observan correlaciones significativas entre la variablel "edad" y las dimensiones "desgaste emocional" e "incompetencia", así como entre "tiempo de actuación en emergencia" y "desarrollo de la carreira". Las primeras están correlacionadas directamente (positivamente) mientras que las dos últimas se correlacionan inversamente (negativamente).

\begin{tabular}{|c|c|c|c|}
\hline Escala de conversão & Idade & $\begin{array}{l}\text { Tempo } \\
\text { Profissão }\end{array}$ & $\begin{array}{l}\text { Atuação em } \\
\text { Emergência }\end{array}$ \\
\hline Desgaste Emocional & $0,675^{\star \star}$ & 0,196 & 0,217 \\
\hline Despersonalização & 0,139 & 0,234 & 0,211 \\
\hline Incompetência & $0,366^{*}$ & 0,203 & 0,275 \\
\hline Ambigüidade de Funções & $-0,166$ & $-0,078$ & $-0,297$ \\
\hline Estrutura Organizacional & $-0,083$ & $-0,157$ & $-0,156$ \\
\hline $\begin{array}{l}\text { Sobrecarga de Trabalho } \\
\text { (quantitativo) }\end{array}$ & 0,145 & 0,104 & 0,065 \\
\hline $\begin{array}{l}\text { Sobrecarga de Trabalho } \\
\text { (qualitativo) }\end{array}$ & $-0,133$ & $-0,130$ & $-0,319$ \\
\hline Desenvolvimento da Carreira & $-0,253$ & $-0,202$ & $-0,445^{\star \star}$ \\
\hline Relações Interpessoais & 0,144 & 0,068 & 0,055 \\
\hline
\end{tabular}

Correlação de Pearson; *Correlação significativa $(p<0,05){ }^{* *}$ Correlação significativa $(p<0,01)$.

La correlación entre las variables "tiempo de actuación en emergencia" y "desarrollo de la carrera" son inversamente proporcionales, esto es, cuanto mayor es el tiempo de actividad profesional menores son las perspectivas de desarrollo de la carrera.

Finalizando la presentación de los resultados, la Tabla 5 muestra la correlación entre las variables de la escala de conversión del $\mathrm{MBI}$, en la que se observa la existencia de correlación significativa entre Desgaste Emocional e Incompetencia; Despersonalización 
con Incompetencia y Desarrollo de la Carrera; Incompetencia con Estructura Organizacional, Desarrollo de la Carrera y Relaciones Interpersonales; Estructura Organizacional con Sobrecarga de Trabajo (cuantitativo), Sobrecarga de Trabajo (cualitativo) y Relaciones Interpersonales; Sobrecarga de Trabajo (cuantitativo) con Relaciones Interpersonales. Las correlaciones mencionadas son directas ylo positivas, lo que significa que la puntuación de las diferentes escalas relacionadas sigue la misma dirección, o sea, el profesional que presenta síntomas de Burnout en una de las escalas sentirá en proporciones semejantes en las demás.

\begin{tabular}{l|cccccccccc}
\multicolumn{7}{c}{ Tabela 5- Correlação entre as variáveis da escala de conversão do } \\
Inventário de Burnout de Maslach \\
\hline Variáveis & DE & DESP & INC & AF & EO & STQT & STQL & DC & RI \\
\hline DE & 1,000 & 0,259 & $0,541^{* *}$ & 0,002 & 0,226 & 0,069 & 0,130 & $-0,287$ & 0,233 \\
& & 1,000 & $0,351^{*}$ & 0,162 & 0,168 & $-0,190$ & 0,143 & - & 0,132 \\
DESP & & & & & & & & $0,332^{*}$ & \\
& & & 1,000 & 0,025 & $0,366^{* *}$ & 0,169 & 0,226 & - & $0,540^{* *}$ \\
INC & & & & & & & & $0,410^{*}$ & \\
AF & & & & 1,000 & 0,168 & $-0,139$ & 0,096 & $-0,020$ & 0,136 \\
EO & & & & & 1,000 & $-0,337^{*}$ & $0,473^{* *}$ & $-0,064$ & $0,490^{* *}$ \\
STQT & & & & & & 1,000 & $-0,183$ & 0,030 & 0,000 \\
STQL & & & & & & & 1,000 & 0,050 & $0,400^{*}$ \\
DC & & & & & & & & 1,000 & $-0,279$ \\
RI & & & & & & & & & 1,000 \\
\hline
\end{tabular}

$\mathrm{DE}=$ Desgaste Emocional; DESP=Despersonalização; INC=Incompetência; AF=Ambigüidade de Funções;

$\mathrm{EO}=$ Estrutura Organizacional STQT=Sobrecarga de Trabalho (quantitativo); STQL=Sobrecarga de Trabalho (qualitativo); $\quad \mathrm{DC}=$ Desenvolvimento da Carreira; RI=Relações Interpessoais

Correlação de Pearson; *Correlação significativa $(\mathrm{p}<0,05) * *$ Correlação significativa $(\mathrm{p}<0,01)$

\section{DISCUSIÓN}

Al analizar el sexo de los investigados, se constata predominancia del femenino. Así, Lima et $\mathrm{al}^{8}$ remiten a los resultados de la investigación Taking the Stress out of Work, realizada por la International Stress Management Association (ISMA-BR), en la que concluyen que las mujeres aunque más estresadas viven más y mejor por verbalizar sus emociones, tener mayor concienciación de sus condiciones físicas y emocionales, buscan ayuda ante las primeras señales y síntomas, son disciplinadas en lo que se refiere a prácticas regulares de relajación y practican una creencia religiosa, demostrando más fe que los hombres.

En relación a la edad, se observa un porcentual mayor de jóvenes, que, según Carlotto; Câmara ${ }^{4}$, presentan mayores niveles de Burnout, debido la comprensión irreal respecto de lo que pueden $\mathrm{o}$ no hacer $\mathrm{y}$, por tanto, son frecuentes las frustraciones profesionales. Principalmente los recién formados, necesitan aprender a lidiar con las demandas del trabajo y, por este motivo, puedenc desarrollar mayores niveles de Burnout, o sea, toda situación nueva desencadena inseguridad y se constituye en un agente estresor capaz de contribuir a la aparición del síndrome.

Importa destacar que más de la mitad de la población investigada está casada y tiene hijos. En este contexto, Gomes et all ${ }^{9}$ citan investigación que compara profesionales con diferentes estados civiles, en la que se constata que los casados muestran mayor deseo de abandonar la profesión y mayores problemas de salud física, mientras que los solteros 
manifiestan mayor malestar relacionado con la inestabilidad profesional. Paralelo a esto, la comparación entre profesionales con y sin hijos menores, permitió observar el hecho de que el deseo de abandonar la profesión es inferior en el grupo de profesionales con hijos menores.

En relación con el tiempo de actuación de los profesionales investigados, se observa que la gran mayoría está trabajando en la profesión en hospital, así como en la Unidad de Urgencia, entre 1 (uno) y 11 (once) años. Carlotto; Palazzo ${ }^{10}$ consideran el Síndrome de Burnout un tipo de estrés de carácter duradero vinculado a las situaciones de trabajo, resultando de la constante y repetitiva presión emocional asociada al intenso compromiso con personas por largos períodos de tempo.

Con la intención de analizar las dimensiones del Síndrome de Burnout, se recolectaron los datos referentes al Inventario de Burnout de Maslach (MBI) y es importante mencionar que la dimensión Desgaste Emocional incluye sentimiento de agotamiento de la energía de los recursos emocionales propios, experiencia de estar emocionalmente desgastado debido al contacto diario con personas en función de su trabajo. La dimensión Despersonalización, analiza actitudes negativas, impersonales, escépticas e insensibles para con las personas, especialmente con los usuarios de los servicios prestados por los respectivos profesionales.

Conforme Murofuse; Abranches; Napoleão ${ }^{11}$ tratar a los usuarios, colegas y la organización como objeto, es una característica de la Despersonalización Paralela a las anteriores, la dimensión Incompetencia, se refiere a la percepción de deterioro de la autocompetencia y falta de satisfacción con las propias realizaciones en el trabajo. Los autores se refieren a Incompetencia en el trabajo como la dimensión en la cual existe un sentimiento de inadecuación personal y profesional. El trabajador tiende a autoevaluarse negativamente, lo que acaba afectando su habilidad para la realización y organización del trabajo, erigiéndose en uno de los principales indicativos del Síndrome de Burnout.

En el transcurso del análisis, se constata marcadores reducidos en las tres dimensiones del síndrome lo que evidencia la baja probabilidad de los investigados en desarrollarlo. La evaluación del Síndrome de Burnout se da, según Carlotto; Câmara ${ }^{4}$ de acuerdo con las puntuaciones de cada dimensión. De esta forma, altas puntuaciones de las dimensiones Desgaste Emocional, Despersonalización e Incompetencia son indicativos de Burnout. Los autores destacan la importancia de evaluar el inventario, considerando su característica tridimensional, a fin de manter su perspectiva de síndrome.

En lo que se refiere al análisis de las categorías en el contexto de trabajo, es importante aclarar que el Síndrome de Burnout no es un problema del individuo, sino del ambiente social en el que desempeña sus actividades laborales. Transformaciones importantes han ocurrido en el mundo del trabajo, las nuevas tecnologías, la gestión organizacional, transitoriedad del empleo, entre otras. Se crean nuevas maneras de organizar el trabajo y las relaciones de los individuos con el mismo son más diversas. Responsabilidad por actividades complejas, competitividad, ambigüedad de funciones $X$ demandas, nuevas exigencias de calidad en la ejecución de las tareas, más cualificación y competencia del trabajador. Sin embargo, la estructura de funcionamiento, la forma de interacción en el trabajo, la deshumanización en las relaciones del empleador-trabajador son lo que parece llevar a la persona a desarollar el referido síndrome.

La Ambigüedad de Funciones, en el contexto del trabajo, presenta porcentuales relativamente bajos en el estudio. Según França; Rodrigues ${ }^{12}$, tener claro cuál es su papel en la organización en que trabaja, ayuda a los trabajadores a posicionarse en las diferentes situaciones con más confianza, propicia la elaboración de un concepto sobre sí mismo más consciente y con repercusiones positivas en su quehacer diario. Paralelamente, la Estructura 
Organizacional presenta medias relativamente elevadas, en este sentido, Campos; Costa ${ }^{13}$ afirman que "el concepto de salud organizacional está directamente asociado al equilibrio entre la estructura, el funcionamiento organizacional y el bienestar individual".

En lo que se refiere a Sobrecarga de Trabajo, cuantitativo y cualitativo, se evidencia medias elevadas y limítrofes, respectivamente. Estos datos son preocupantes y merecedores de la atención del gestor de la respectiva unidad, dado que la varible sobrecarga es importante en el diagnóstico dl Burnout, así como en la comprensión de este fenómeno.

La categoría Desarrollo de la Carrera obtuvo media alta y diferentes autores afirman que el Síndrome de Burnout puede ser consecuencia de la falta de oportunidad para el profesional de crecer en su carrera. Así, Dutra ${ }^{14}$ expone que el éxito de la organización está directamente relacionado con su capacidad de desarrollar personas. Este desarrollo debe respetar la individualidad de cada una de ellas. En relación a las Relaciones Interpersonales, esta presenta una elevación benéfica de su média y, de acuerdo con Stumm; Maçalai; Kirchner ${ }^{15}$ el conflicto es inherente a las relaciones interpersonales y no debe ser encarado como negativo, al contrario, precisa ser discutido constructivamente, incitando cambios, oportunizando qué actitudes puedan ser repensadas, buscando nuevas y mejores formas de actuar.

En lo que se refiere al análisis de la correlación entre las variables edad, tiempo de profesión y de actuación en emergencia se constata significante asociación entre la edad y, más específicamente la juventud, con el desgaste emocional y la baja realización profesional. Santos; Alves; Rodrigues ${ }^{16}$ afirman que en lo que se refiere a la edad, la mayoría de los estudios sobre el Burnout, revela que individuos relativamente jóvenes son más propensos al síndrome, ya que las personas más jóvenes teoricamente tienen alta expectativa frente al trabajo, lo que puede llevar a frustraciones cuando no perciben el debido reconocimiento.

Los jóvenes profesionales tienden a sentirse más inseguros en cuanto a sus conocimientos, son más afectados por las reacciones de sus pacientes y, muchas veces, alimentan falsas expectativas sobre sus carreras. Profesionales con más tiempo de profesión, los más viejos, probablemente ya desarrollaron estrategias de coping que les permiten lidiar mejor con las situaciones estresantes derivadas del trabajo.

Paralelo a esto, se observa que cuanto más largo es el tiempo de profesión, menores son las expectativas de éxito en la carrera. El Síndrome de Burnout puede surgir como consecuencia de un desgaste crónico por el tiempo de profesión y, también, debido a la inexperiencia del profesional en el desempeño de las actividades inherentes a su profesión. Algunos autores describen que tras un tiempo mayor de actuación en la profesión, el profesional puede entrar en un periodo de sensibilización, volviéndose más vulnerable. Esa sensibilización se debe al hecho de que, tras ese tiempo de ejercicio profesional, ya hubo la transición de las expectativas idealistas iniciales de la profesión a la práctica cotidiana, percibiéndose que las recompensas personales, profesionales y económicas no son las esperadas $^{16}$.

Jodas; Haddad ${ }^{17}$ resaltan la importancia de que la institución valore y reconozca el trabajo desarrollado, así como invertir e incentivar el desarrollo profesional. Las organizaciones que invierten en el perfeccionamiento de sus colaboradores, tienden a valorarlos como seres humanos. Sin embargo, el trabajador es responsable de la gestión de su desarrollo, de su competitividad profesional, así como de su carrera, de esta forma, necesita ser consciente de su papel y responsabilizarse de la gestión de su carrera, así como exigir de la organización condiciones objetivas para su desarrollo profesional. Estudios muestran que al final de la década de los 90 las personas empezaron a valorar las oportunidades concretas que el mercado de trabajo ofrece ${ }^{14}$. 
En relación a la correlación existente entre las variables Desgaste Emocional e Incompetencia, los autores Tironi et all $^{18}$ señalan que una vez que el profesional se siente ineficiente, con disminución de la autoconfianza y sensación de fracaso, ocurre reducción en la realización personal en el trabajo. La ineficacia durante la realización de las actividades se torna predominante, en este caso, importa destacar que esta dimensión se considera la última reacción al estrés generado por las exigencias del trabajo. Añadir que el síndrome causa la pérdida de la relación de sentido de ese profesional y su trabajo, surgiendo un sentimiento de apatía y desánimo y, como consecuencia, la dificultad de vislumbrar estrategias que retomen la motivación, el placer y la salud. Paralelamente, las autoras Jodas; Haddad ${ }^{17}$ afirman que las situaciones de difícil recuperación o de no recuperación del enfermo, encontradas por profesionales, pueden llevar a un sentimiento de gran insatisfacción profesional. La falta de preparación para enfrentar la muerte también puede generar un sentimiento de impotencia.

La Enfermería fue clasificada por la Health Education Authority como la cuarta profesión más estresante. Además, encuentra dificultades en delimitar los diferentes papeles de la profesión y, consecuentemente, la falta de reconocimiento nítido, elevando la despersonalización del trabajador en relación a la profesión ${ }^{11}$. Cabe destacar algunas actitudes, tales como, el deterioro en la calidad de servicios de instituciones de salud y los altos índices de absentísmo de los profesionales de esta área como algunas de las consecuencias de este cuadro de despersonalización, baja realización profesional y déficit en el desarrollo de la carrera. La despersonalización, según Carlotto; Palazzo ${ }^{10}$ induce al profesional a cuestionamientos sobre hasta que punto su trabajo es factor de realización y satisfacción. Las afirmativas de los autores mencionados justifican la correlación existente entre las variables Despersonalización, Incompetencia y Desarrollo de la Carrera.

En lo que atañe a la correlación entre las variables Incompetencia, Estructura Organizacional, Desarrollo de la Carrera y Relaciones Interpersonales, Tamayo; Tróccoli ${ }^{19}$ dicen que los procesos de gestión y de administración, o sea, las variables de apoyo organizacional pueden reducir significativamente este sentimento en los trabajadores. Para que esto ocurra, según ellos, es importante que estos procesos sean construídos colectivamente, de manera participativa, incentivando y valorando el trabajo del equipo. Para los autores, el agotamiento puede ser reducido igualmente mejorando las relaciones interpersonales en el trabajo, incluyendo colegas, supervisores y regulación del proceso de trabajo de la organización. Esto puede ser viable por medio de políticas claras, incluyendo planificación, actualización, información y consiguiente implementación de políticas de ascenso, promoción y salarios, contribuyendo a la satisfacción de los profesionales en su carrera. Murofuse; Abranches; Napoleão ${ }^{11}$ se refieren a la incompetencia en el trabajo como una dimensión en la cual existe un sentimiento de inadecuación personal y profesional. El trabajador tiende a autoevaluarse negativamente, lo que acaba afectando su habilidad para la realización del trabajo, el contacto con los usuarios del servicio, así como con la organización.

Las variables Sobrecarga de Trabajo Cualitativo y Relaciones Interpersonales están igualmente correlacionadas en este estudio. Referente a las mismas, Maslach; Leiter ${ }^{20}$ indican que el exceso de trabajo puede ser la indicación más precisa de desequilibrio entre el trabajador y la institución en la que trabaja. "Hemos de hacer mucho, en poco tiempo y con pocos recursos. No se trata de dar de sí para enfrentar nuevos desafíos, sino de ir más allá de los límites humanos" (p.26). Los autores mencionan que el exceso de trabajo aumenta, concomitantemente, con el ritmo del trabajo, interferiendo en la calidad de la asistencia, en las relaciones con colegas y contribuyendo al desgaste físico y emocional del trabajador. Ellos se refieren a las expectativas y deseos de los trabajadores, importantes y que interfieren en el proceso de trabajo, inclusive, en los resultados. 
Se constata correlación entre las variables Sobrecarga de Trabajo Cuantitativo y Estructura Organizacional. En este sentido, cabe destacar que el número reducido de profesionales está directamente relacionado con el exceso de trabajo. Además, la insatisfacción con la organización de la empresa, así como con el salario agrava la situación, llevando a los profesionales a tener otro vínculo en su relación de trabajo con el consecuente aumento en la carga horaria mensual. ${ }^{11}$

Desde el punto de vista organizacional, el profesional en estado de Burnout puede presentar consecuencias en el proceso de trabajo, afectando la calidad de asistencia de enfermería prestada. Se observa que la dinámica organizacional del trabajo en una Unidad de Urgencia genera sobrecarga de movimiento y tensión ocupacional, siendo necesario monitorizar periódicamente la salud mental y física de los trabajadores, a fin de desarrollar estrategias que reorganicen el proceso de trabajo disminuyendo las fuentes de estrés. ${ }^{17}$

Ante lo expuesto, destaca la importancia de que el trabajador desarrolle estrategias individuales y colectivas, para preservar su salud, incluso enfrentando las más diversas presiones y dificultades en el ambiente de trabajo.

\section{CONCLUSIONES}

Las transformaciones en el mundo del trabajo interfieren tanto en el lugar de trabajo como en las relaciones sociales del trabajador, pudiendo causar daños al bienestar físico y emocional del mismo. Se alcanzó el objetivo de caracterizar al equipo de profesionales de la salud que actúa en una Unidad de Urgencia de un hospital porte IV del noroeste de Rio Grande do Sul, así como correlacionar las variables del Síndrome de Burnout.

A partir de los instrumentos de análisis se constató que los profesionales estudiados vivencian numerosos estresores en el trabajo, sin embargo, los resultados muestran bajo nivel de Burnout. Finalizando, la toma de actitudes en pro de la salud de los trabajadores es vital, considerando que las transformaciones técnicas y organizacionales introducidas en los procesos de trabajo han provocado acentuado desgaste en los mismos.

De esta forma, se concluye que, bajo ciertas condiciones, el trabajo puede contribuir a la enfermedad. Sin embargo, muchas situaciones de insatisfacción profesional pueden solucionarse por acciones simples, o sea, el equipo actúa en un espacio común, se dedica a objetivos comunes, de esta manera, el proceso de transformación necesita ocurrir colectivamente, buscando oportunidades de crecimiento y satisfacción para que este equipo continúe actuando con compromiso, responsabilidad, conciencia crítica y se mantenga saludable.

\section{REFERENCIAS}

1. Saviani D. Trabalho e educação: fundamentos ontológicos e históricos. Revista Brasileira de Educação [online]. 2007, vol.12, n.34, pp. 152-165. ISSN 1413-2478. Disponível em: http://www.scielo.br/pdf/rbedu/v12n34/a12v1234.pdf. Acesso em 29 de maio de 2010.

2. Barros DS. Médicos plantonistas de unidade de terapia intensiva: perfil sócio-demográfico, condições de trabalho e fatores associados à síndrome de Burnout. Revista Brasileira de Terapia Intensiva [online]. 2008, vol.20, n.3, pp. 235-240. ISSN 0103-507X. doi: 10.1590/S0103-507X2008000300005. Disponível em: http://www.scielo.br/pdf/rbti/v20n3/v20n3a05.pdf. Acesso em 29 de maio de 2010.

3. Martínez OF, Cabrera CH, Tapia AM, Suárez SM, Garcia BGDR. Burnout among resident physicians Who work duty shifts in the emergency department. Emergências. 2007; 19:116- 
21. Disponível em: http://www.semes.org/revista/vol19_3/3_ing.pdf. Acesso em 02 de junho de 2010.

4. Carlotto MS, Câmara SG. Análise da produção científica sobre a Síndrome de Burnout no Brasil. Revista Psico. Porto Alegre, PUCRS, v. 39, n. 2, pp. 152-158, abr./jun. 2008.

Disponível em:

http://revistaseletronicas.pucrs.br/ojs/index.php/revistapsico/article/viewFile/1461/3035.

Acesso em 30 de maio de 2010.

5. Ritter RS, Stumm EMF, Kirchner RM. Análise de Burnout em profissionais de uma unidade de emergência de um hospital geral. Revista Eletrônica de Enfermagem [Internet]. 2009; 11(2):236-48. Disponível em: http://www.fen.ufg.br/revista/v11/n2/v11n2a02.htm. Acesso em 30 de maio de 2010.

6. Feliciano KVO, Kovacs MH, Sarinho SW. Sentimentos de profissionais dos serviços de pronto-socorro pediátrico: reflexões sobre Burnout. Revista Brasileira Saúde Materno Infantil, Recife, v. 5, n. 3, p. 319-27, jul./set 2005. Disponível em: http://www.scielo.br/pdf/rbsmi/v5n3/a08v5n3.pdf. Acesso em 29 de maio de 2010.

7. Lautert L. O desgaste profissional do enfermeiro. [tese] Salamanca (Espanha): Universidade Pontífica de Salamanca; 1995.

8. Lima FD et al. Síndrome de Burnout em residentes da Universidade Federal de Uberlândia - 2004. Revista Brasileira de Educação Med. [online]. 2007, vol.31, n.2, pp. 137-146. ISSN 0100-5502. Disponível em: http://www.scielo.br/pdf/rbem/v31n2/03.pdf. Acesso em 02 de junho de 2010.

9. Gomes R et al. Stresse, "Burnout”, saúde física, satisfação e realização em profissionais de saúde: análise das diferenças em função do sexo, estado civil e agregado familiar. Braga : Universidade do Minho, Portugal, 2008. vol. 4, p. 178-192. Disponível em: http://repositorium.sdum.uminho.pt/handle/1822/7824. Acesso em 02 de junho de 2010.

10. Carlotto MS, Palazzo LS. Síndrome de Burnout e fatores associados: um estudo epidemiológico com professores. Caderno de Saúde Pública [online]. 2006, vol.22, n.5, pp. 1017-1026. ISSN 0102-311X. Disponível em: http://www.scielosp.org/pdf/csp/v22n5/14.pdf. Acesso em 02 de junho de 2010.

11. Murofuse NT; Abranches SS, Napoleão AA. Reflexões sobre estresse e burnout e a relação com a enfermagem. Revista Latino-Americana de Enfermagem, 2005, mar./abr, v. 13, n.2, p.255-61. Disponível em: http://www.scielo.br/pdf/rlae/v13n2a19.pdf. Acesso em 01 de maio de 2007.

12. França ACL, Rodrigues AL. Stress e trabalho: uma abordagem psicossomática. Ed.3. São Paulo: ATLAS, 2002.

13. Campos ICM, Costa FN. Cultura e saúde nas organizações. Estudos da Psicologia, Campinas/SP, 2007, abr./jun., v.24, n.2, p.279-282. Disponível em:

http://www.scielo.br/pdf/estpsi/v24n2/v24n2a14.pdf. Acesso em 08 de setembro de 2007.

14. Dutra JS. Gestão de pessoas: modelo, processos, tendências e perspectivas. ed.1. reimp. 5. São Paulo: Atlas, 2006.

15. Stumm EMF, Maçalai RT, Kirchner RM. Dificuldades enfrentadas por enfermeiros em um centro cirúrgico. Texto \& Contexto Enfermagem. Florianópolis/ SC, v. 15, n.003, p. 464471, julh./set., 2006. Disponível em: http://redalyc.uaemex.mx/redalyc/pdf/714/71415311.pdf. Acesso em 20 de novembro de 2007. 
16. Santos FE, Alves JA, Rodrigues AB. Síndrome de Burnout em enfermeiros atuantes em uma Unidade de Terapia Intensiva. Revista Einstein. 2009; 7(1 Pt 1):58-63. Disponível em: http://apps.einstein.br/revista/arquivos/PDF/979-Einsteinv7n1p58_63.pdf. Acesso em 20 de junho de 2010.

17. Jodas DA, Haddad MCL. Síndrome de Burnout em trabalhadores de enfermagem de um pronto socorro de hospital universitário. Acta paul. enferm. [online]. 2009, vol.22, n.2, pp. 192-197. ISSN 0103-2100. Disponível em: http://www.scielo.br/pdf/ape/v22n2/a12v22n2.pdf. Acesso em 19 de junho de 2010.

18. Tironi MOS et al. Trabalho e síndrome da estafa profissional (Síndrome de Burnout) em médicos intensivistas de Salvador. Revista Associação Medica Brasileira [online]. 2009, vol.55, n.6, pp. 656-662. ISSN 0104-4230. Disponível em:

http://www.scielo.br/pdf/ramb/v55n6/09.pdf. Acesso em 19 de junho de 2010.

19. Tamayo MR, Tróccoli BT. Exaustão emocional: relações com a percepção de suporte organizacional e com as estratégias de coping no trabalho. Estudos de Psicologia, Brasília, v. 7, n. 1, p. 37-46, 2002. Disponível em: http://www.scielo.br/pdf/epsic/v7n1/10952.pdf. Acesso em: 16 de setembro de 2007.

20. Maslach C, Leiter MP. Trabalho: fonte de prazer ou desgaste? Guia para vencer o estresse na empresa. Campinas, SP: Papirus, 1999. 